\title{
Microstructural Behaviors of Matrices Based on Polylactic Acid and ${ }_{2}$ Polyhydroxyalkanoates
}

\author{
${ }_{3}$ Juan Carlos Alzate Marin, ${ }^{\dagger}$ Sandra Rivero, ${ }^{*},+, \oplus \odot$ Adriana Pinotti, ${ }^{\dagger}{ }^{\dagger}$ Alejandro Caravelli, $^{\dagger}$ \\ 4 and Noemí Elisabet Zaritzky, \\ $5{ }^{\dagger}$ Centro de Investigación y Desarrollo en Criotecnología de Alimentos (CIDCA), CONICET, Universidad Nacional de La Plata \\ 6 (UNLP), Comisión de Investigaciones Científicas de la Provincia de Buenos Aires (CICPBA), 47 y 116 S/N, La Plata B1900AJJ, \\ 7 Buenos Aires, Argentina \\ $8{ }^{\star}$ Facultad de Ciencias Exactas, Universidad Nacional de La Plata (UNLP), La Plata B1900AJJ, Buenos Aires, Argentina \\ $9{ }^{\S}$ Facultad de Ingeniería, Universidad Nacional de La Plata (UNLP), La Plata B1900AJJ, Buenos Aires, Argentina
}

10 ABSTRACT: Individual films of polyhydroxyalkanoates (PHA) and polylactic acid (PLA) and their blends were developed by 11 solvent casting. PHA was obtained from activated sludges from a wastewater-treatment system at a laboratory scale. This work 12 focused on analyzing the microstructural properties and thermal behaviors of individual films of PHA and PLA as well as those 13 of their blends. The behaviors of the biodegradation processes of the individual films and blends were examined from a 14 microstructural point of view. ATR-FTIR spectra indicated the existence of weak molecular interactions between the polymers.

15 The formulation of blend films improved the crystallinity of PLA; additionally, it induced the polymer-recrystallization 16 phenomenon, because crystallized PHA acted as a PLA-nucleating agent. This phenomenon explains the improvements in the 17 films' water-vapor-barrier properties. The blends exposed to a biodegradation process showed an intermediate behavior between 18 PLA and PHA, leading to a consistent basis for designing systems tailored to a particular purpose.

19 KEYWORDS: biopolymers, polymer blends, infrared spectroscopy, crystallinity, modulated DSC, morphology

\section{$20 \quad$ INTRODUCTION}

21 Polyhydroxylalkanoate (PHA) is a family of biopolyesters 22 synthesized by microorganisms from various carbon sources; 23 they are accumulated intracellularly under nutrient stress and 24 act as carbon and energy reserves. ${ }^{1}$ PHAs can be produced by 25 both pure and mixed microbial cultures. Mixed cultures have 26 the advantage that they do not need sterile conditions and are 27 better able to adapt to complex substrates, such as industrial 28 wastes, than pure cultures. ${ }^{2}$ For this, wastes from the dairy 29 industry, the sugar industry, forestry, and biodiesel production 30 can be utilized. ${ }^{3}$ Cheese whey is a surplus material from the 31 dairy industry that is mainly discharged to both soils and water 32 bodies, causing serious environmental problems. PHA 33 production from cheese whey could revalorize this dairy 34 subproduct and, at the same time, solve the waste-disposal 35 problems.

36 A relevant characteristic of PHA is its versatility, because 37 there are more than a hundred different monomers, including 38 hydroxyvalerate and butyrate among others. The properties of 39 the synthesized polymer are modified depending on the 40 position of its functional groups and degrees of polymerization. 41 This chemistry allows PHAs to be tailored to provide similar 42 properties to traditional thermoplastics, such as polyethylene 43 (PE) and polypropylene (PP), while maintaining biodegrad44 ability. ${ }^{4}$

45 Polylactic acid (PLA), a thermoplastic aliphatic polyester 46 derived from lactic acid (2-hydroxy propionic acid), can be 47 produced by condensation and polymerization directly from its 48 basic building block, lactic acid, which is derived from the 49 fermentation of sugars from carbohydrates sources, such as corn starch; cassava root, chips, and starch; and sugar cane. ${ }^{5} 50$ Polylactic acid is produced at the largest industrial scale of all 51 biodegradable polymers, and it is considered the most 52 promising candidate for replacing conventional plastics. ${ }^{6}$ It is 53 being used in biomedical applications, for bottle production, 54 for filament production in 3D printing, and for compostable- 55 food-packaging production. It is also being evaluated as a 56 material for tissue engineering. Mass production has reduced 57 the cost of PLA production, making it an economically viable 58 choice for the fabrication of containers, plastic bags, and fibers. 59 Commercial-scale plants today produce hundreds of thousands 60 of tons of PLA per year.?

By controlling the molecular architecture, suppliers are able 62 to tailor the polymer to specific applications. Consequently, 63 new and improved grades of bioplastic resins are constantly 64 being introduced to the market. NatureWorks LLC focuses on 65 a multistep procedure involving the condensation reaction of 66 aqueous lactic acid for the production of a low-molecular- 67 weight PLA prepolymer. It is converted into a blend of lactide 68 stereoisomers in order to produce a large spectrum of PLA 69 grades. ${ }^{8}$ The films based on PLA have excellent optics and 70 good machinability, barrier, and mechanical properties. ${ }^{5} \quad 71$

PLA and PHA polymers are polyesters and are used in 72 consumer products by a wide industrial sector as a result of 73 their biocompatibility and sustainability. ${ }^{9}$ They have thermal 74

Received: March 25, 2018

Revised: July 14, 2018

Accepted: July 23, 2018

Published: July 23, 2018 
75 behaviors comparable to those of some conventional polymers, 76 and this has generated much interest in exploring their physical 77 and structural properties to identify potential applications. ${ }^{10}$

78 The combination of the two polymers allows the design of 79 new materials with tailorable properties differing meaningfully 80 from those of each component by adjusting the advantages of 81 each polymer in order to obtain materials for different 82 applications. In this sense, the formulation of blend systems 83 is easier and faster than copolymerization methods. ${ }^{11}$ There 84 are many studies focused on PLA modification, such as the 85 addition of modifiers and copolymerization. ${ }^{12}$ The addition of 86 a highly ordered stereochemical structure, such as that of PHA, 87 could improve the film properties of PLA. ${ }^{13,14}$ In this sense, 88 blending PLA with another biobased and biodegradable 89 material, PHA, in order to obtain tailor-made materials 90 constitutes a promising alternative.

91 This work focused on analyzing the microstructural 92 properties and thermal behaviors of PHA and PLA individual 93 films as well as those of their blends. Furthermore, the 94 biodegradation processes of the individual films and PLA/PHA 95 blends were examined from a microstructural point of view.

\section{MATERIALS AND METHODS}

97 Polylactic acid (PLA), grade 4043D in pellet form (98\% L-lactide with 98 a D-isomer content of approximately $2 \%$ ) and designed for the 99 production of films, was purchased from Natureworks under the 100 trademark Ingeo. As is well-known, the production of PLA involves 101 the conversion of starch in dextrose via a hydrolysis process and 102 fermentation by microorganisms to synthesize the chain of the 103 polylactide polymer.

104 Polyhydroxylalkanoate (PHA) was obtained from activated sludges 105 from a wastewater-treatment system at the laboratory scale (CIDCA). 106 A two-stage biological system was proposed to produce PHA by 107 aerobic mixed culture from hydrolyzed cheese whey. The first step 108 involved the selection of PHA-accumulating organisms using an 109 aerobic sequencing batch reactor (SBR) operated under a feast110 famine regime. Then, there was the stage of PHA accumulation in an 111 aerobic batch reactor that was inoculated with biomass taken from 112 SBR enriched with PHA-accumulating bacteria.

113 Extraction and Quantification of PHA. Extraction and 114 characterization of PHA from the batch-reactor biomass were 115 performed following the method proposed by Venkateswar Reddy 116 et al. $^{15}$ A hydroxybutyrate-hydroxyvalerate (HB-HV) copolymer 117 with a purity of $96 \%$ and a molar relation $95: 5 \mathrm{HB}-\mathrm{HV}$ was obtained. 118 Film Preparation. The films were prepared by dissolving PHA 119 and PLA in chloroform at 1 and $2 \%(\mathrm{w} / \mathrm{v})$, respectively, under stirring 120 for $3 \mathrm{~h}$. PLA/PHA mixtures were prepared in the following 121 proportions: 20/80, 40/60, 60/40, and 80/20 (w/w). They were 122 stirred for $30 \mathrm{~min}$ at $60^{\circ} \mathrm{C}$.

123 Filmogenic solutions of PLA, PHA, and their blends were cast into 124 glass Petri dishes $(9 \mathrm{~cm}$ diameter) that were left under a fume hood. 125 Later, the samples were dried in a vacuum oven at $60{ }^{\circ} \mathrm{C}$ to ensure 126 solvent removal from the matrices. When the solutions were cast, 127 constant mass-molding-area ratios were maintained in order to 128 ensure uniform thicknesses in the different samples, because the 129 control solutions (PLA and PHA) had different concentrations.

130 Film thickness was measured by means of a coating-thickness gauge 131 (Check Line DCN-900) for nonconductive materials on nonferrous 132 substrates. For each specimen, at least 14 measurements at different 133 positions were taken.

134 Water-Vapor Permeability. Water-vapor-permeability (WVP) 135 tests were carried out according to the technique described by Rivero 136 et al. $^{16}$ based on a modified version of ASTM ${ }^{17}$ method E96. A 137 permeation cell maintained at $20{ }^{\circ} \mathrm{C}$ was used for the assays. Eight 138 measurements were performed once steady-state conditions were 139 reached. All measurements were performed at least four times for each 140 specimen.
Thermal Analysis by MDSC. Thermal analysis of the samples 141 was conducted by the modulated-differential-scanning-calorimetry 142 (MDSC) technique. Q100 (TA Instruments) controlled by a TA 143 5000 module (TA Instruments) and equipped with a cryogenic- 144 quench-cooling accessory was used under a $\mathrm{N}_{2}$ atmosphere. This 145 technique allowed us to measure the calorific capacity of the sample 146 and simultaneously differentiate between the reversible (dependent 147 on the calorific capacity) and irreversible events (dependent on the 148 time and temperature) that the sample experienced. Likewise, it 149 allowed the resolution of complex transitions.

A standard heating ramp of $10{ }^{\circ} \mathrm{C} \mathrm{min}^{-1}$ with a modulation- 151 temperature amplitude of $0.5{ }^{\circ} \mathrm{C}$ and a modulation period of $60 \mathrm{~s}$ was 152 used. The first scan (heating) was performed in order to delete the 153 thermal story from $-50{ }^{\circ} \mathrm{C}$ up to $200{ }^{\circ} \mathrm{C}$. Once the first scan was 154 completed, the sample was cooled to $-80{ }^{\circ} \mathrm{C}$, and then a second 155 heating scan was recorded. Informed results were the averages of two 156 replicates to ensure reproducibility of the results.

157

The results were analyzed by using the Universal Analysis V1.7F 158 software (TA Instruments). Different parameters were determined 159 from the obtained thermograms: melting temperature $\left(T_{\mathrm{m}}\right)$, enthalpy 160 corresponding to the area of the endothermic peak $\left(\Delta H_{\mathrm{m}}\right), 161$ crystallization temperature during the cooling stage $\left(T_{c}\right)$, crystal- 162 lization enthalpy $\left(\Delta H_{\mathrm{c}}\right)$, and glass-transition temperature $\left(T_{\mathrm{g}}\right) 163$ determined from the reversible signal.

164

ATR-FTIR Spectroscopy. The interactions between the system 165 components were analyzed using the ATR-FTIR technique. Spectra of 166 the samples were registered by using the Thermo Scientific Nicolet 167 iS10 FT-IR Spectrometer in the wavenumber region of 4000-400 168 $\mathrm{cm}^{-1}$, performing 64 scans at a $4 \mathrm{~cm}^{-1}$ resolution. Specimens were 169 placed onto the diamond ATR crystal (Smart iTX accessory), and the 170 software Omnic 8 (Thermo Scientific) was used for the data-analysis 171 process. To ensure the reproducibility of the results, the tests were 172 performed in triplicate.

X-ray Diffraction. X-ray diffractograms of individual PVA and 174 PLA films and their blends were evaluated by using an X'Pert Pro P 175 Analytical Model PW 3040/60 (Almelo) operated at room temper- 176 ature. The $\mathrm{Cu} \mathrm{K} \alpha$ radiation (1.542 $\AA$ ) was generated at $30 \mathrm{~mA}$ and 40177 $\mathrm{kV}$. Scattered radiation was detected in an angular range of $3-60^{\circ} 178$ $(2 \theta)$ at a step size of $0.02^{\circ}$. The crystallinity degree (CD) was 179 calculated following the procedure described in previous works. ${ }^{18,19} 180$

Structural Studies by SEM. The microstructures of the matrices 181 were examined by using an FEI model Quanta 200 scanning electron 182 microscope. Individual films and blend matrices were immersed in 183 liquid nitrogen and fractured cryogenically. Samples were mounted 184 onto metal stubs by using double-sided tape and observed at low 185 pressure and an acceleration voltage of $12.5 \mathrm{kV}$, without any metal or 186 carbon coating.

Biodegradation Test. A series of plastic pots $\left(400 \mathrm{~cm}^{3}\right)$ were 188 used as soil containers, and the microflora present was used as a 189 degrading medium. Square specimens of $3 \times 3 \mathrm{~cm}$ with a thickness of 190 approximately $25 \mu \mathrm{m}$ on average were put into a plastic mesh to allow 191 the access of moisture and microorganisms and the retrieval of 192 degraded specimens. In order to guarantee aerobic degradation, the 193 film samples were buried. The samples were conditioned at a relative 194 humidity of $50 \%$ and a temperature of $20{ }^{\circ} \mathrm{C}$. Containers were 195 sprayed regularly with a defined and constant amount of water. 196 Similar conditions were employed by Rivero et al. ${ }^{20}$ At specific time 197 intervals, specimens were recovered from the soil and cleaned with a 198 brush to avoid any possible damage of the structure.

In accordance with the described methodology in ASTM D5988- 200 $03,{ }^{21}$ a ratio of $1 \mathrm{~g}$ of compost per $25 \mathrm{~g}$ of soil was used. Studies of the 201 morphology of the degraded films were performed by means of SEM, 202 MDSC, and ATR-FTIR analysis.

Statistical Analysis. For statistical analysis, version 10.0 of Systat- 204 software (Systat, Inc.) was used. Analysis of variance (ANOVA), 205 linear regressions, and Fisher's least-significant-difference test for 206 mean comparison were performed. The significance level used was 207 0.05 . 
Thermal Analysis. The thermograms obtained by means 211 of MDSC from PLA, PHA, and their blends can be observed in ${ }_{212}$ Figure 1, where the stages of heating and cooling are shown. 213 The temperatures of the thermal transitions and the enthalpy 214 values $\left(\mathrm{J} \mathrm{g}^{-1}\right)$ of the characteristic events during the heating
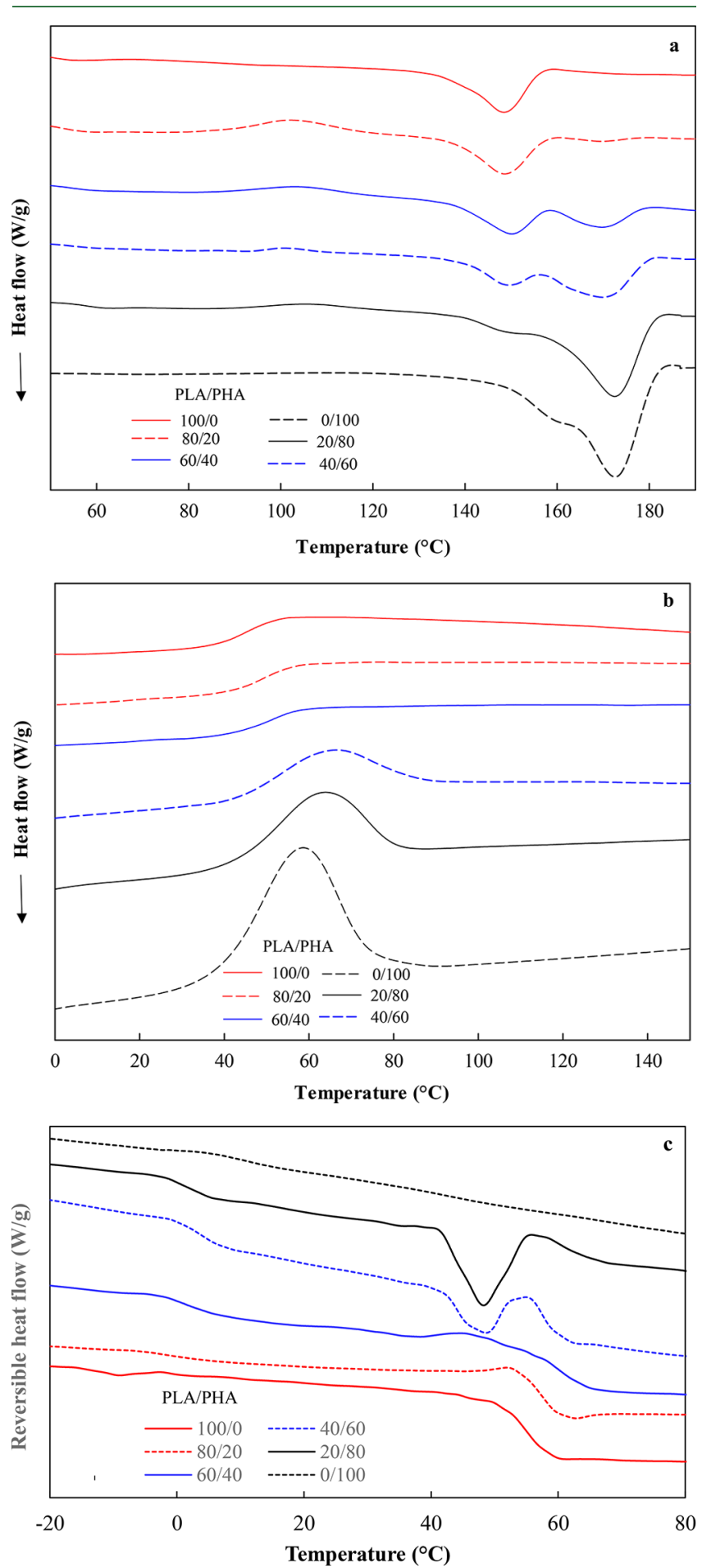

Figure 1. MDSC curves of individual (PLA and PHA) and blend films with different proportions of PLA/PHA showing (a) heating stage, (b) cooling stage, and (c) reversing heat flow $\left(\mathrm{W} \mathrm{g}^{-1}\right)$, evidencing the glass-transition temperatures $\left(T_{\mathrm{g}}\right)$ of each component. and cooling stages obtained from MDSC curves are $215 \mathrm{t} 1$ summarized in Table 1.

As it is possible to see in Figure 1a, the thermal analysis 217 allowed us to observe a peak for each individual film, 218 attributable to the melting of the crystalline domains 219 corresponding to PLA and PHA at 147.7 and $172.7{ }^{\circ} \mathrm{C}$, with 220 the associated enthalpies of 20.1 and $86.6 \mathrm{~J} \mathrm{~g}^{-1}$, respectively. 221 The intensity of the melting peak is related to the number of 222 crystals or crystalline populations (crystalline fraction) that 223 exist in the material when the melting occurs. Using MDSC, 224 Thellen et al. ${ }^{4}$ reported that crystal melting is produced at 225 higher temperatures in samples of PHA containing lower 226 percentages of valerate, because as the valerate content 227 increases, the onset of crystal melting occurs at lower 228 temperatures. In the present study, the PHA thermograms 229 evidenced the presence of a peak with a shoulder, which could 230 represent the melting of crystal lamellae, as was reported by 231 Thellen et al. ${ }^{4}$ for extruded PHA.

232

On the other hand, PHA films exhibited a $T_{\mathrm{g}}$ at $-4.7^{\circ} \mathrm{C}, 233$ whereas PLA showed a marked transition at $58.1{ }^{\circ} \mathrm{C}$ (Figure 234 1c). Glass-transition temperatures ranging from 2.7 to $-4.4{ }^{\circ} \mathrm{C} 235$ for a PHA matrix obtained by casting were reported by da Silva 236 et al. ${ }^{22}$

The thermograms of all the mixtures exhibited PLA- 238 recrystallization peaks at $107{ }^{\circ} \mathrm{C}$; this transition was more 239 evident with higher PLA proportions in the mixture (Figure 240 1a). These results indicate that the incorporation of PHA 241 contributes to the process of recrystallization of PLA in all the 242 blend formulations. This effect was more marked in the 80/20 243 blend, a behavior that could be explained by bearing in mind 244 that a low proportion of crystalline domains of PHA dispersed 245 in a continuous matrix of PLA induced the phenomenon of 246 nucleation of this latter polymer. The presence of double 247 melting temperatures in the PLA/PHA blends corresponds to 248 the melting of both the "as formed" and recrystallized 249 polymer. $^{10}$

According to Abdelwahab et al. ${ }^{10}$ and Ikehara et al., ${ }^{23}{ }_{251}^{250}$ recrystallization strongly depends on the melting-temperature 252 differences among the components of the mixture. When the 253 $T_{\mathrm{m}}$ difference is very large, the component with the higher 254 melting temperature crystallizes first, and its spherulites 255 contribute to fill all the volume.

The $20 / 80$ blend exhibited a peak at $171.9{ }^{\circ} \mathrm{C}$ because of the 257 melting of the PHA crystalline fraction and a shoulder at 148.4258 ${ }^{\circ} \mathrm{C}$ corresponding to the melting of PLA. The 80/20, 60/40, 259 and 40/60 blends exhibited two endothermic transitions; the 260 enthalpy associated with the first event diminished with the 261 incorporation of a higher proportion of PHA to the blend, 262 whereas the enthalpy of the endothermic peak, characteristic of 263 the PHA-crystalline phase, became more prominent (Figure 1a 264 and Table 1). These findings were in accordance with a higher 265 crystalline degree.

From the obtained DSC curves, two $T_{\mathrm{g}}$ values were observed 267 irrespective of the blend composition. These results suggest 268 that the analyzed PLA/PHA blends are immiscible in the 269 amorphous state (Figure 1c).

According to Lipatov and Alekseeva, ${ }^{24}$ the appearance of 271 two transitions associated with the glass-transition temper- 272 atures supports the existence of a partially miscible system (i.e., 273 materials with a heterogeneous biphasic structure).

274

During the cooling step, PHA exhibited a peak of 275 crystallization at $58.7^{\circ} \mathrm{C}$ with an associated enthalpy of 48.4276 $\mathrm{J} \mathrm{g}^{-1}$, whereas the thermogram of PLA did not show this 277 
Table 1. Thermal Analysis of Single and Blend Matrices with Different Proportions of PLA/PHA ${ }^{a}$

\begin{tabular}{|c|c|c|c|c|c|c|}
\hline \multirow[b]{3}{*}{ PLA/PHA } & \multicolumn{4}{|c|}{ heating stage } & \multirow{2}{*}{\multicolumn{2}{|c|}{$\begin{array}{c}\text { cooling stage } \\
\text { characteristic events of PHA }\end{array}$}} \\
\hline & \multicolumn{2}{|c|}{ characteristic events of PHA } & \multicolumn{2}{|c|}{ characteristic events of PLA } & & \\
\hline & $T_{\mathrm{m}}\left({ }^{\circ} \mathrm{C}\right)$ & $\Delta H_{\mathrm{m}}\left(\mathrm{J} \mathrm{g}^{-1}\right)$ & $T_{\mathrm{m}}\left({ }^{\circ} \mathrm{C}\right)$ & $\Delta H_{\mathrm{m}}\left(\mathrm{J} \mathrm{g}^{-1}\right)$ & $T_{\mathrm{c}}\left({ }^{\circ} \mathrm{C}\right)$ & $\Delta H_{c}\left(\mathrm{~J} \mathrm{~g}^{-1}\right)$ \\
\hline $100 / 0$ & - & - & $147.7(1.0) \mathrm{a}$ & $20.1(1.3) \mathrm{c}$ & - & - \\
\hline $80 / 20$ & $170.4(1.2) \mathrm{a}$ & $1.5(0.07) \mathrm{a}$ & $150.3(1.9) \mathrm{a}$ & $20.0(0.7) \mathrm{c}$ & - & - \\
\hline $60 / 40$ & $169.9(0.6) \mathrm{a}$ & $9.1(0.1) \mathrm{b}$ & $149.8(0.6) \mathrm{a}$ & $13.4(0.5) \mathrm{b}$ & - & - \\
\hline $40 / 60$ & $170.3(0.4) \mathrm{a}$ & $18.8(0.7) \mathrm{c}$ & $149.5(0.9) \mathrm{a}$ & $5.8(0.1) \mathrm{a}$ & $65.6(0.5) \mathrm{b}$ & $18.8(0.1)$ a \\
\hline $20 / 80$ & $171.9(0.8) \mathrm{a}$ & $61.7(1.2) \mathrm{d}$ & - & - & $65.9(3.8) \mathrm{b}$ & $31.4(1.9) \mathrm{b}$ \\
\hline $0 / 100$ & $172.7(0.1) \mathrm{a}$ & $86.6(0.1) \mathrm{e}$ & - & - & $58.8(0.5)$ a & $48.4(0.4) \mathrm{c}$ \\
\hline
\end{tabular}

${ }^{a}$ Different letters in the same column indicate significant differences $(p<0.05)$ among samples.

278 transition (Figure $1 \mathrm{~b}$ and Table 1 ). Taking these results into 279 account, it is possible to infer that the crystallinity of PHA is 280 greater than that of PLA.

281 The thermal analysis of the PLA/PHA mixtures showed that 282 the peak of crystallization attributable to PHA was observed 283 only in the $20 / 80$ and $40 / 60$ mixtures, with a minor associated 284 enthalpy regarding a single PHA matrix (Figure $1 \mathrm{~b}$ ). On the 285 other hand, the thermograms of the individual PLA film and 286 the $60 / 40$ and $80 / 20$ mixtures did not exhibit this thermal 287 transition (Figure 1b). Nevertheless, an event was observed at $28857.7{ }^{\circ} \mathrm{C}$ attributable to the glass-transition temperature of the 289 PLA-enriched phase. Similar results were informed by Arrieta 290 et al., ${ }^{12}$ Furukawa et al., ${ }^{25}$ and Zhang and Thomas, ${ }^{11}$ indicating 291 that these results are a signal of the fact that the PHA in the 292 mixtures does not crystallize during the cooling stage when it is 293 the minor proportion.

294 Microstructural Analysis. FTIR Analysis. From the ATR295 FTIR spectra, the functional characteristic groups of PLA and 296 PHA were identified (Figure 2a,b). They are consistent with 297 those reported in the literature. ${ }^{5,26,27}$

298 In the PLA spectrum, the band located at $1746 \mathrm{~cm}^{-1}$ is a 299 strong peak that corresponds to the stretching of the carbonyls' 300 amorphous phase. ${ }^{5}$ The bands located at 1180 and $1080 \mathrm{~cm}^{-1}$ 301 belong to the asymmetric and symmetric stretching vibrations 302 of $\mathrm{C}-\mathrm{O}-\mathrm{C}$. As is known, PLA is a hydrophobic polymer 303 because of the presence of $-\mathrm{CH}$ side groups. The peaks at 304 about 2997 and $2946 \mathrm{~cm}^{-1}$ correspond to the asymmetric and 305 symmetric stretching vibrations of the $-\mathrm{CH}$ groups in the side 306 chains, respectively (data not shown).

307 On the other hand, in the PHA spectrum, the peak at 1719 $308 \mathrm{~cm}^{-1}$ is attributable to the stretching of the crystalline carbonyl 309 group, and the stretching of the carbonyl group of the 310 amorphous phase is virtually imperceptible. The 40/60 and 311 20/80 samples exhibited peaks at 1748 and $1755 \mathrm{~cm}^{-1}$ (Figure $312 \mathrm{2b}$ ), which were assigned to the amorphous and crystalline 313 bands of PLA, respectively. ${ }^{24}$

314 Furthermore, Figure 2a,b depicts the ATR-FTIR spectra of 315 the PLA/PHA blend matrices. The spectra of the 80/20 and $31660 / 40$ compositions followed a similar pattern to that of the 317 pristine PLA, whereas the 40/60 and 20/80 mixtures were 318 identified mainly with the PHA spectrum. All of them unfolded 319 both bands corresponding to the stretching of carbonyls 320 located at 1746 and $1719 \mathrm{~cm}^{-1}$ due to PLA and PHA phase, 321 respectively (Figure 2a,b).

322 In the cases of the $80 / 20,60 / 40$, and $40 / 60$ blends, the 323 bands in the $\mathrm{C}-\mathrm{O}-\mathrm{C}$ and $\mathrm{C}-\mathrm{C}$ stretching and $\mathrm{CH}$ 324 deformation mode region $\left(1300-1000 \mathrm{~cm}^{-1}\right)$ are visualized 325 at about the same wavenumbers as those of pure PHA (Figure $3262 a, b)$.
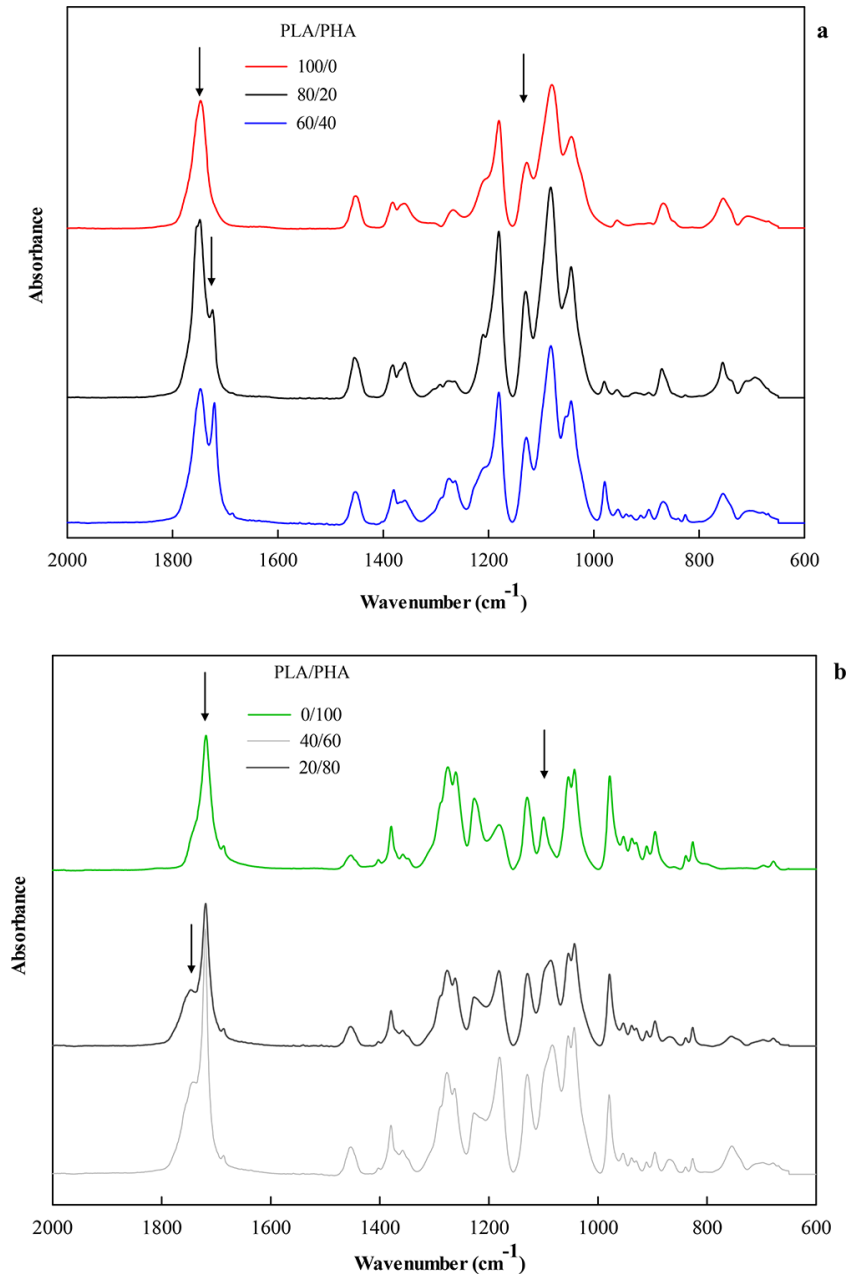

Figure 2. ATR-FTIR spectra of individual (PLA and PHA) and blend matrices with different proportions of PLA/PHA.

It is noteworthy that the intensities of these bands were 327 interdependent and mutually dependent, one being increased 328 at the expense of the other, meaning that the ratio of intensities 329 of these two bands changed in agreement with the ratio of the 330 individual components. These results allowed us to infer the 331 immiscibility of these components and that there were no 332 strong molecular interactions between the polymers. 333

$X$-ray Diffraction. The typical diffraction patterns of 334 individual PHA and PLA and those of the composite films 335 are depicted in Figure 3. The X-ray diffractogram of the PLA $336 \mathrm{f} 3$ films exhibited a pattern corresponding to an amorphous 337 material with a peak located at $2 \theta=17^{\circ}$, which was associated 338 with the reflection of the crystals of the polymer; ${ }^{13,28}$ the 339 


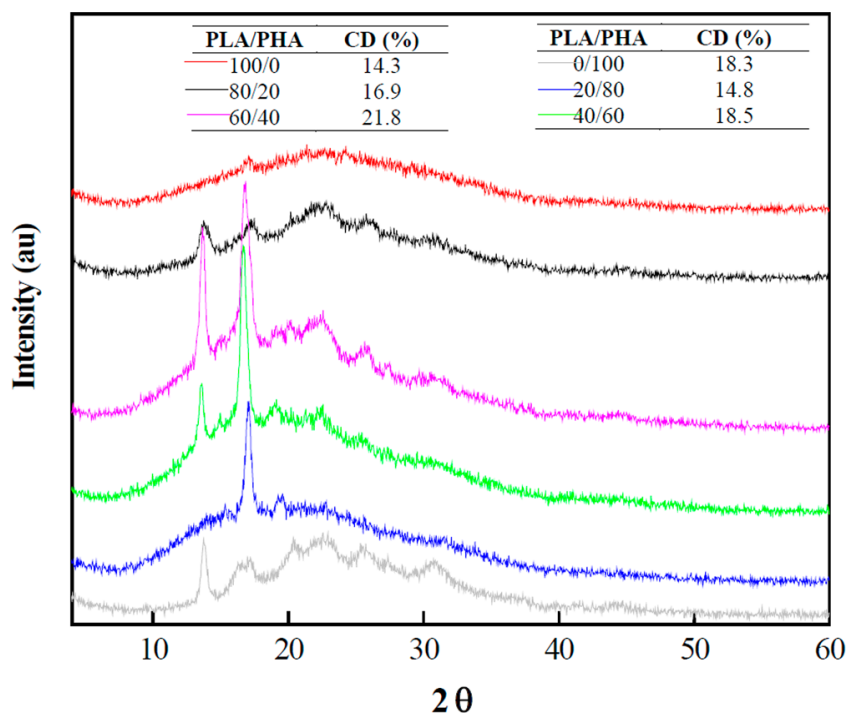

Figure 3. X-ray diffractograms of individual (PLA and PHA) and blend films with different proportions of PLA/PHA. Table inset shows the crystallinity degrees (\%) of the samples.

340 estimated crystallinity degree was $14.3 \%$ (table insert in Figure $3413)$. In contrast, PHA is a highly ordered polymer, and it is 342 known to crystallize in an orthorhombic cell. ${ }^{10}$

343 X-ray-diffraction analysis was used to determine the 344 crystalline structures and crystallinity degrees of the blends. 345 The PHA spectrum showed diffraction peaks at $2 \theta=13.8$ and $34617^{\circ}$, corresponding to the (020) and (110) planes, 347 respectively. $^{11}$ Additionally, reflections at $2 \theta=20.3,22.4$,
25.4, and $30.8^{\circ}$ were detected, which were characteristics of 348 the PHA polymer, its CD being $18.3 \% .^{14}$

The inclusion of PHA in the blends increased the 350 crystallinity of the materials. The highest crystallinity degrees 351 of the $60 / 40$ and $40 / 60$ blends were confirmed by means of X- 352 rays (table insert in Figure 3 ). The diffractograms showed 353 peaks of higher intensity in relation to individual matrices. 354

This behavior allows us to infer that PHA induces the 355 recrystallization of PLA due to its capacity to act as an agent of 356 nucleation. According to Furukawa et al. ${ }^{24}$ and Zhang and 357 Thomas, ${ }^{11}$ it generates better packing density of the polymeric 358 segments and promotes better adhesion and interaction at the 359 interface level. However, the microstructure, porosity, and 360 permeability of the matrix seem to depend on the PLA/PHA 361 proportions, as presented below.

362

SEM Observations. From the macroscopic point of view, 363 the blend of the two polymers appeared to be well mixed in the 364 matrices with no apparent phase separation, although FTIR 365 showed the immiscibility of both components. The average 366 thickness of the films was $25 \mu \mathrm{m}$, which was corroborated by 367 microscopic analysis. As it can be seen in Figure 4, all matrices $368 \mathrm{f} 4$ presented homogeneous appearances irrespective of the PLA/ 369 PHA ratio.

The films showed important differences when analyzed in 371 the cross-sections of single or blend matrices. PLA-only films 372 showed a homogeneous appearance, without pores and with 373 good structural integrity. Addition of the PHA polymer led to 374 films whose cross-section was rough, giving a structure with a 375 fibrous appearance (Figure 4).

The cross-sections and surfaces of the 20/80 and 40/60 377 mixtures revealed the presence of an orderly structure, 378 attributable to the growth and crystallization of the spherulites 379
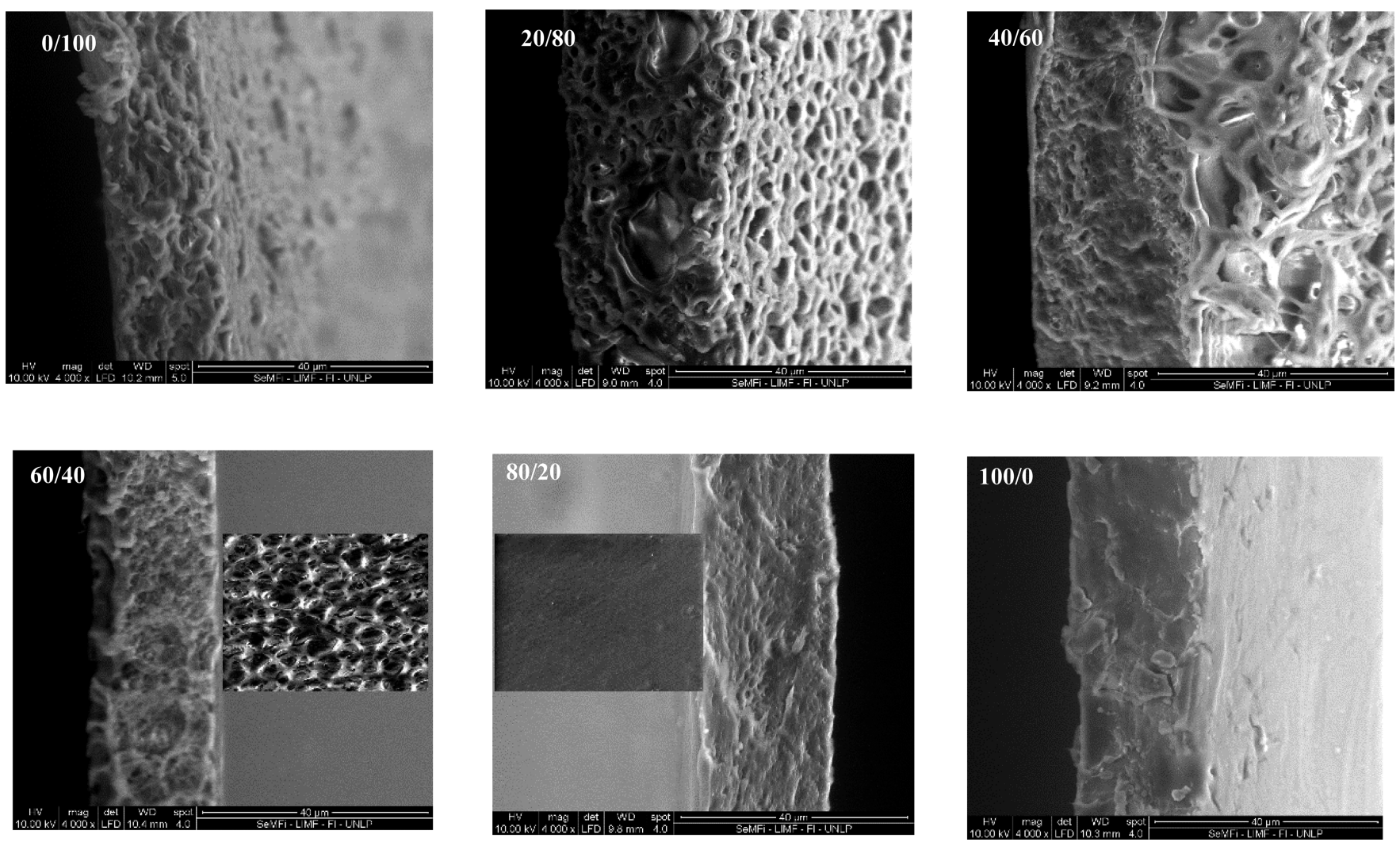

Figure 4. Cross-section SEM micrographs of individual (PLA and PHA) and blend films with different proportions of PLA/PHA. 
380 of PHA, proving to be a network formed by codomains 381 corresponding to the different polymers with a granular, 382 interspersed appearance and a repetitive pattern. It is 383 important to remark that the highest proportion of PHA in 384 the blends $(\mathrm{PLA} / \mathrm{PHA}=20 / 80)$ evidenced a porous 385 microstructure as a result of the structural arrangement 386 between the polymers. According to Abdelwahab et al., ${ }^{10}$ the 387 interface interactions between the PHA and PLA phases could 388 influence the nucleation phenomenon as supported by the 389 XRD results.

390 These micrographs reinforced the results of immiscibility 391 obtained by other techniques. As the PLA proportion 392 increased, the morphology of the matrices became more 393 homogeneous and less rough, until the 80/20 mixture 394 presented an appearance where the PLA-enriched phase 395 formed a continuous domain in which the PHA-enriched 396 phase was dispersed and homogeneously distributed, as can be 397 seen in Figure 4.

398 Water-Vapor Permeability. Table 2 shows the WVP values 399 of the individual films and PLA/PHA blends. The formulation

Table 2. Water-Vapor-Barrier Properties of the Individual (PLA and PHA) and Blend Films with Different Proportions of PLA/PHA ${ }^{a}$

$\begin{array}{cc}\text { PLA/PHA } & \text { WVP }\left(\times 10^{11} \mathrm{~g} \mathrm{~s}^{-1} \mathrm{~m}^{-1} \mathrm{~Pa}^{-1}\right) \\ 100 / 0 & 1.73(0.25) \mathrm{b} \\ 80 / 20 & 0.62(0.06) \mathrm{a} \\ 60 / 40 & 0.68(0.07) \mathrm{a} \\ 40 / 60 & 0.72(0.05) \mathrm{a} \\ 20 / 80 & 2.22(0.38) \mathrm{b} \\ 0 / 100 & 2.24(0.30) \mathrm{b}\end{array}$

${ }^{a}$ Different letters indicate significant differences $(p<0.05)$ among samples.

400 of the blend matrices improved the barrier properties of the 401 materials, obtaining a reduction of $40 \%$ in the WVP values for 402 the $40 / 60,60 / 40$, and $80 / 20$ blends compared with the values 403 of the individual films (Table 2). Meanwhile, the 20/80 blend 404 did not show significant differences $(p>0.05)$.

405 According to Arrieta et al., ${ }^{12}$ the crystallization of PLA 406 reduces the water-vapor permeability because the crystals 407 decrease the volume of the amorphous phase, generating a 408 path of greater tortuosity and reducing mass matter transfer.

409 The improvement of the barrier properties is related to the 410 higher crystallinity of the PLA/PHA blend matrices, as 411 observed by the X-ray diffraction technique and the MDSC 412 thermal analysis.

413 Soil-Biodegradation Studies. The influence of the micro414 structure developed during the formulation of the blends on 415 the biodegradation process was examined. A morphological 416 study by SEM during soil-biodegradation experiments was 417 complementary to visual examination as it helped to confirm 418 the existence of structural modifications in the films, allowing a 419 detailed evaluation of the degradation process.

420 The results showed that the degradation behavior of the 421 biobased polymers followed different patterns regarding the 422 morphological characteristic of each matrix. As can be seen in 423 Figure 5a, the biodegradation occurred in both the PHA and 424 PLA phases.

425 The biodegradation assay revealed that the PHA films 426 exhibited remarkable biodegradation after 30 days, whereas the 427 blend matrices (PLA/PHA) showed changes around 50 days, which indicated a delay in the process of biodegradation 428 (Figure 5a). According to Weng et al., ${ }^{27}$ a PHA polymer could 429 be biodegraded at a high rate under composting as well as soil- 430 environment conditions; in contrast, although PLA was 431 biodegradable under composting conditions, its degradation 432 rate in soil was slow.

433

SEM micrographs show the fractured surface of the PLA/ 434 PHA matrices after a biodegradation process of 50 days 435 (Figure 5a). It can be seen that the biodegradation was 436 intensified with higher proportions of PHA in the blend 437 system. As the proportion of PHA increased, particularly from 438 $40 / 60$ to $20 / 80$, the matrices showed greater interstices or 439 cavities (Figure 4), which could facilitate the biodegradation 440 process. Zhang and Thomas ${ }^{11}$ explained that the inclusion of 441 PHA improved the degradation degree of PLA at room 442 temperature. The authors reported that PHA and PLA exhibit 443 different degradation patterns. PHA is mainly degraded by the 444 attack of various enzymes at the surface. According to Weng et 445 $\mathrm{al}^{27}$ the degradation of PHA can be attributed to erosion 446 catalyzed by bacteria from the surface to the interior. 447

In contrast, PLA degradation is produced throughout the 448 whole sample, starting with a nonenzymatic hydrolysis that 449 leads to a reduction in the molecular weight. ${ }^{11}$ Then, low- 450 molecular-weight PLA diffuses out of the bulk polymer and can 451 be metabolized by microorganisms, producing water, carbon 452 dioxide, and humus. ${ }^{29}$

On the other hand, the thermograms of the 20/80, 40/60 454 and $60 / 40$ blend matrices exposed to the biodegradation 455 process for 50 days showed a decrease of the transition 456 enthalpy, corresponding to the melting of the PHA crystalline 457 fraction being more marked in the blends with the greatest 458 proportion of PHA (Figure 5b). In this sense, Dharmalingam ${ }^{30} 459$ found that the changes of melting enthalpy $\left(\Delta H_{\mathrm{m}}\right)$ for a 460 composite material based on PLA/PHA blends represent 461 depolymerization. Consequently, this phenomenon could 462 indicate a higher sensitivity to the degradation process in the 463 presence of higher proportions of PHA, independent of the 464 rearrangement formed between both polymers. 465

The chemical-structure changes of the matrices exposed to 466 the biodegradation process were investigated by ATR-FTIR 467 (Figure 5c).

ATR-FTIR spectra corroborated the changes caused in the 469 structure of the polymers. After 50 days of exposure to the 470 biodegradation process, ATR-FTIR spectra of the PHA and 471 blend films showed that the bands lost definition. 472

For all the samples assayed, the peaks in the 4000-3000 473 $\mathrm{cm}^{-1}$ region became broadened after the biodegradation 474 process (data not shown). According to Weng et al., ${ }^{27}$ this 475 fact was attributable to the formation of hydroxyl and 476 carboxylic groups. This was also confirmed by the shift of 477 the absorption peak of the $\mathrm{C}=\mathrm{O}$ stretching vibration after 478 biodegradation.

Ludueña et al. ${ }^{31}$ pointed out that chemical and enzymatic 480 hydrolysis are the main mechanisms of chain rupture during 481 the degradation process. Then, biodegradation process 482 depends on water availability, which produces hydrolysis, and 483 microbial attack of the matrix. Water diffusion in the soil leads 484 to a hydrolytic degradation of the material modifying the 485 surface, resulting in a porous structure with evident signs of 486 degradation. A similar explanation was proposed by Rocca- 487 Smith et al. ${ }^{32}$ 

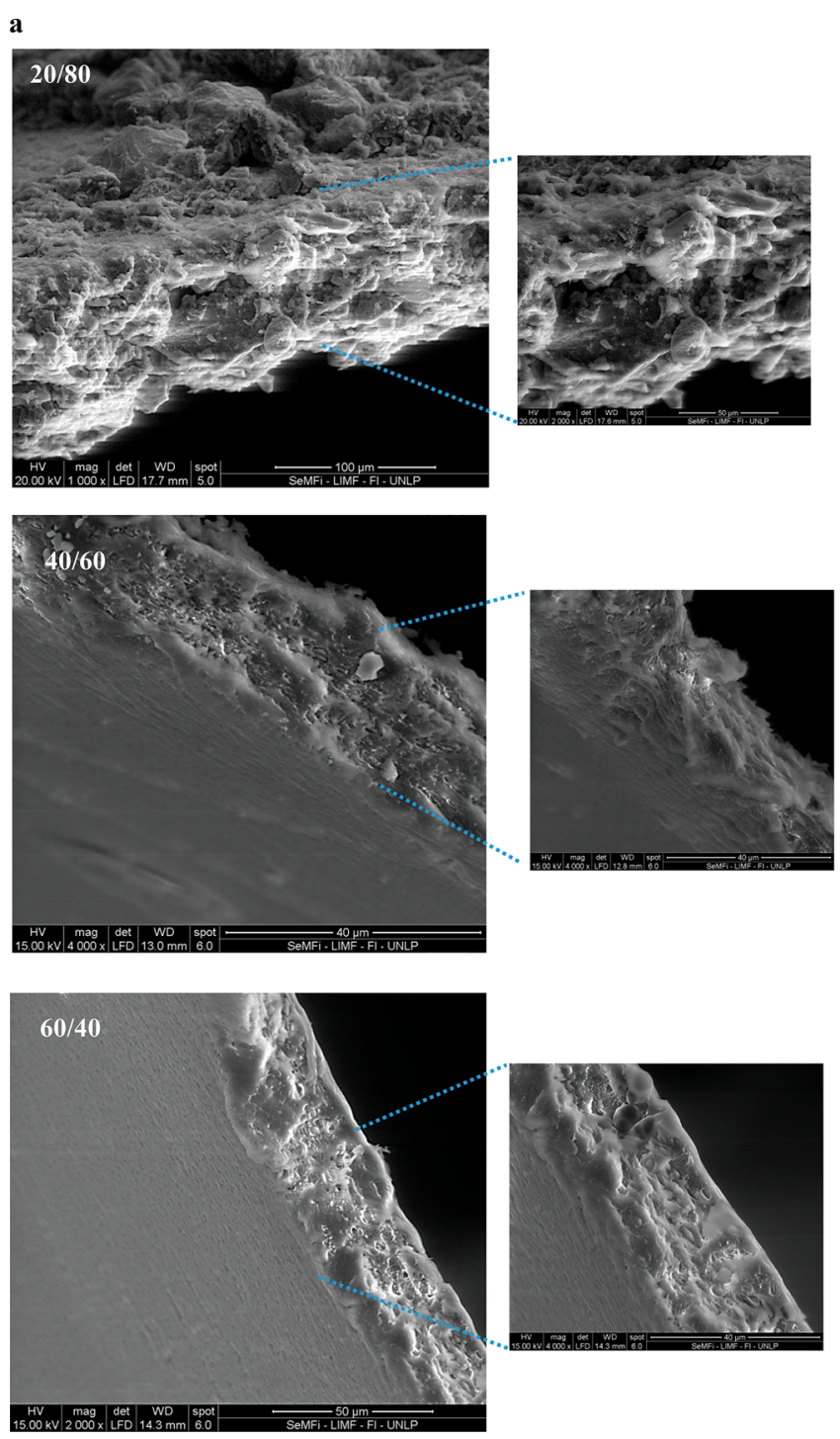

Figure 5. Monitoring of the behaviors of samples after they were submitted to biodegradation processes (buried for 50 days) through (a) SEM micrographs of the PLA/PHA blend matrices with enlarged sections showing the degradation (magnification is indicated in the micrographs), (b) MDSC analysis, and (b) FTIR-ATR studies.

489 The biodegradability of the blends was more marked in films 490 formulated with increasing proportions of PHA; similar results 491 were reported by Zhang and Thomas. ${ }^{11}$

492 Conclusions. Thermal and microstructural studies revealed 493 that blends of PLA/PHA-based systems were partially miscible. 494 The formulation of blend matrices improved the crystallinity of 495 PLA; additionally, it induced the polymer-recrystallization 496 process, because crystallized PHA acted as a PLA-nucleating 497 agent. This phenomenon explains the improvements in water498 vapor-barrier film properties. Moreover, the blends exposed to 499 a biodegradation process showed an intermediate behavior 500 between those of PLA and PHA, leading to a consistent basis 501 for designing systems tailored to a particular purpose or use. 502 This work, therefore, contributes to the knowledge of 503 microstructures, allowing one to develop and test materials 504 with specific technological applications.

\section{AUTHOR INFORMATION}

\section{Corresponding Author}

507 *E-mail: sandra_gmr@yahoo.com. Tel. and Fax: +54 0221508 424-9287, +54 0221-425-4853, or +54 0221-489-0741.
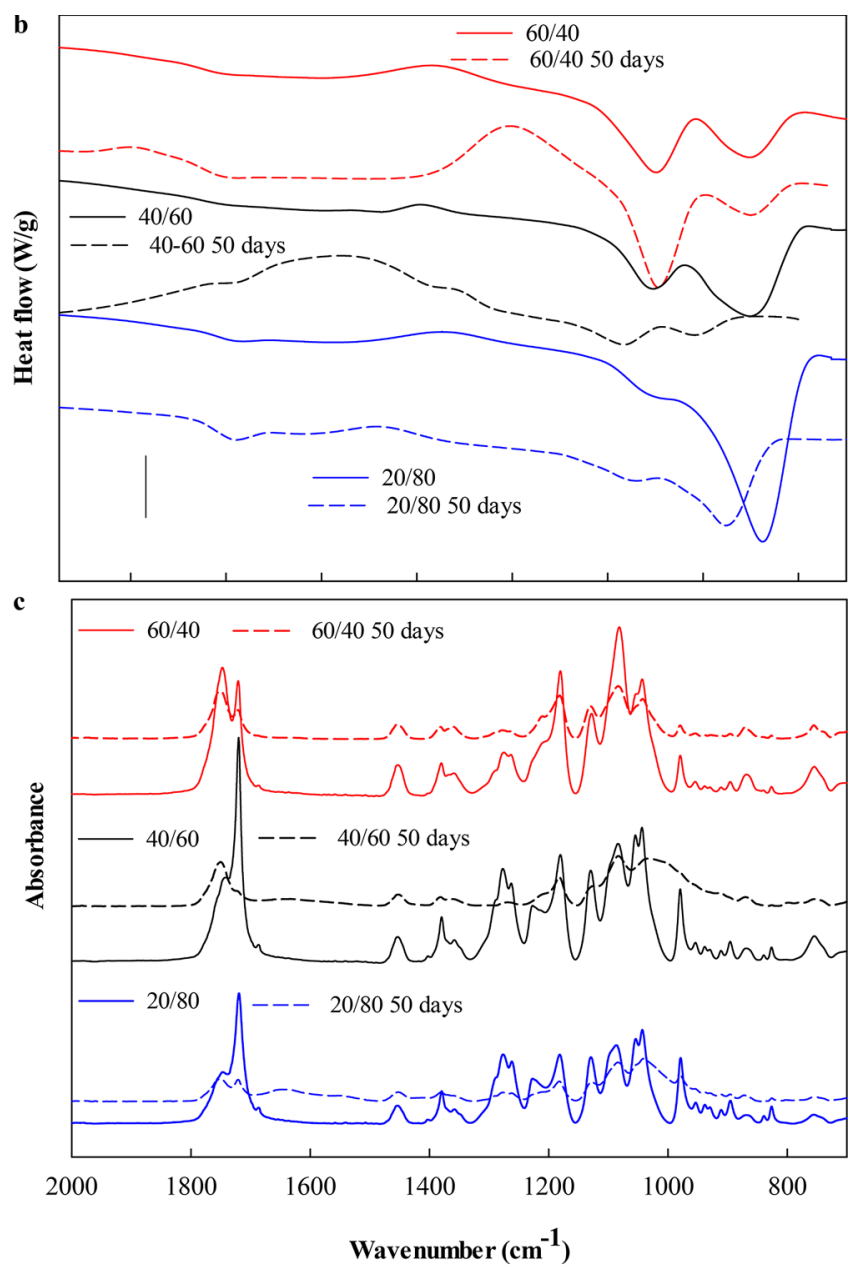

ORCID

Sandra Rivero: 0000-0002-9002-182X 510

Funding

511

This work was supported by the Argentinean Agency for the 512 Scientific and Technological Promotion (ANPCyT; Project 513 PICT 2012-0415, 2014-1620), the Argentinean National 514 Research Council (CONICET, PIP 2013-0109), and the 515 University of La Plata (Argentina).

Notes

The authors declare no competing financial interest.

\section{ACKNOWLEDGMENTS}

The authors acknowledge Ing. Javier Lecot, Lic. Diana Velasco, 520 and Daniel Russo for technical assistance.

\section{REFERENCES}

(1) Ren, J. Biodegradable Poly (Lactic Acid): Synthesis, Modification, 523 Processing; Springer-Verlag: Berlin, 2011.

(2) Colombo, B.; Sciarria, T. P.; Reis, M.; Scaglia, B.; Adani, F. 525 Polyhydroxyalkanoates (PHAs) production from fermented cheese 526 
527 whey by using a mixed microbial culture. Bioresour. Technol. 2016, 528 218, 692-699.

529 (3) Mothes, G.; Schnorpfeil, C.; Ackermann, J.-U. Production of 530 PHB from crude glycerol. Eng. Life Sci. 2007, 7, 475-479.

531 (4) Thellen, C.; Coyne, M.; Froio, D.; Auerbach, M.; Wirsen, C.; 532 Ratto, J. A. A processing, characterization and marine biodegradation 533 study of melt-extruded polyhydroxyalkanoate (PHA) films. J. Polym. 534 Environ. 2008, 16 (1), 1-11.

535 (5) Rivero, S.; Lecot, J.; Pinotti, A. Impregnation of kraft paper 536 support with polylactic acid multilayers. Adv. Mater. Lett. 2017, 8 (6), $537741-751$.

538 (6) Madhavan Nampoothiri, K.; Nair, N. R.; John, R. P. An overview 539 of the recent developments in polylactide (PLA) research. Bioresour. 540 Technol. 2010, 101 (22), 8493-8501.

541 (7) Sin, L. T. Polylactic Acid: PLA Biopolymer Technology and 542 Applications, 1st ed.; Elsevier, 2012.

543 (8) Murariu, M.; Dubois, P. PLA composites: From production to 544 properties. Adv. Drug Delivery Rev. 2016, 107, 17-46.

545 (9) Armentano, I.; Fortunati, E.; Burgos, N.; Dominici, F.; Luzi, F.; 546 Fiori, S.; Jiménez, A.; Yoon, K.; Ahn, J.; Kang, S.; Kenny, J. M. 547 Processing and characterization of plasticized PLA/PHB blends for 548 biodegradable multiphase systems. eXPRESS Polym. Lett. 2015, 9 (7), 549 583-596.

550 (10) Abdelwahab, M. A.; Flynn, A.; Chiou, B. S.; Imam, S.; Orts, W.; 551 Chiellini, E. Thermal, mechanical and morphological characterization 552 of plasticized PLA-PHB blends. Polym. Degrad. Stab. 2012, 97 (9), $553 \quad 1822-1828$.

554 (11) Zhang, M.; Thomas, N. L. Blending polylactic acid with 555 polyhydroxybutyrate: the effect on thermal, mechanical, and 556 biodegradation properties. Adv. Polym. Technol. 2011, 30 (2), 67-79. 557 (12) Arrieta, M. P.; López, J.; Hernández, A.; Rayón, E. Ternary 558 PLA-PHB-Limonene blends intended for biodegradable food 559 packaging applications. Eur. Polym. J. 2014, 50, 255-270.

560 (13) Arrieta, M. P.; Castro-Lopez, M. D. M.; Rayón, E.; Barral561 Losada, L. F.; López-Vilariño, J. M.; López, J.; González-Rodríguez, 562 M. V. Plasticized poly (lactic acid)-poly (hydroxybutyrate)(PLA$563 \mathrm{PHB}$ ) blends incorporated with catechin intended for active food564 packaging applications. J. Agric. Food Chem. 2014, 62 (41), 1017056510180 .

566 (14) Arrieta, M. P.; Fortunati, E.; Dominici, F.; López, J.; Kenny, J. $567 \mathrm{M}$. Bionanocomposite films based on plasticized PLA-PHB/cellulose 568 nanocrystal blends. Carbohydr. Polym. 2015, 121, 265-275.

569 (15) Venkateswar Reddy, M.; Nikhil, G. N.; Venkata Mohan, S.; 570 Swamy, Y. V.; Sarma, P. N. Pseudomonas otitidis as a potential 571 biocatalyst for polyhydroxyalkanoates (PHA) synthesis using 572 synthetic wastewater and acidogenic effluents. Bioresour. Technol. 573 2012, 123, 471-479.

574 (16) Rivero, S.; García, M. A.; Pinotti, A. Crosslinking capacity of 575 tannic acid in plasticized chitosan films. Carbohydr. Polym. 2010, 82 576 (2), 270-276.

577 (17) Standard test methods for water vapor transmission of material. 578 In Annual book of ASTM, E96-95; American Society for Testing and 579 Materials: Philadelphia, PA, 1995.

580 (18) Lamarra, J.; Giannuzzi, L.; Rivero, S.; Pinotti, A. Assembly of 581 chitosan support matrix with gallic acid-functionalized nanoparticles. 582 Mater. Sci. Eng., C 2017, 79, 848-859.

583 (19) Villarruel, S.; Giannuzzi, L.; Rivero, S.; Pinotti, A. Changes 584 induced by UV radiation in the presence of sodium benzoate in films 585 formulated with polyvinyl alcohol and carboxymethyl cellulose. Mater. 586 Sci. Eng., C 2015, 56, 545-554.

587 (20) Rivero, S.; García, M. A.; Pinotti, A. Physical and chemical 588 treatments on chitosan matrix to modify film properties and kinetics 589 of biodegradation. J. Mater. Physics Chem. 2013, 1 (3), 51-57.

590 (21) Standard Test Method for determining aerobic biodegradation 591 in soil of plastic materials or residual plastic materials after 592 composting. In Annual book of ASTM, D5988-03; American Society 593 for Testing and Materials: Philadelphia, PA, 2003.

594 (22) da Silva, M. G. D.; Vargas, H.; Poley, L. H.; Rodriguez, R. S.; 595 Baptista, G. B. Structural impact of hydroxyvalerate in polyhydrox- yalkanoates (PHAscl) dense film monitored by XPS and photo- 596 thermal methods. J. Braz. Chem. Soc. 2005, 16 (4), 790-795. 597

(23) Ikehara, T.; Kimura, H.; Qiu, Z. Penetrating spherulitic growth 598 in poly (butylene adipate-co-butylene succinate)/poly (ethylene 599 oxide) blends. Macromolecules 2005, 38 (12), 5104-5108. 600

(24) Lipatov, Y. S.; Alekseeva, T. Phase-Separated Interpenetrating 601 Polymer Networks; Springer-Verlag: Berlin, 2007.

(25) Furukawa, T.; Sato, H.; Murakami, R.; Zhang, J.; Duan, Y. X.; 603 Noda, I.; Ochiai, S.; Ozaki, Y. Structure, dispersibility, and crystallinity 604 of poly (hydroxybutyrate)/poly (L-lactic acid) blends studied by FT- 605 IR microspectroscopy and differential scanning calorimetry. Macro- 606 molecules 2005, 38 (15), 6445-6454.

(26) Ashok, B.; Naresh, S.; Reddy, K. O.; Madhukar, K.; Cai, J.; 608 Zhang, L.; Rajulu, A. V. Tensile and Thermal Properties of Poly(lactic 609 acid)/Eggshell Powder Composite Films. Int. J. Polym. Anal. Charact. 610 2014, 19, 245-255.

611

(27) Weng, Y. X.; Wang, L.; Zhang, M.; Wang, X. L.; Wang, Y. Z. 612 Biodegradation behavior of $\mathrm{P}(3 \mathrm{HB}, 4 \mathrm{HB}) / \mathrm{PLA}$ blends in real soil 613 environments. Polym. Test. 2013, 32 (1), 60-70.

(28) Reis, M. O.; Olivato, J. B.; Bilck, A. P.; Zanela, J.; Grossmann, 615 M. V. E.; Yamashita, F. Biodegradable trays of thermoplastic starch/ 616 poly (lactic acid) coated with beeswax. Ind. Crops Prod. 2018, 112, 617 481-487.

(29) Auras, R.; Harte, B.; Selke, S. An Overview of polylactides as 619 packaging materials. Macromol. Biosci. 2004, 4, 835-864. 620

(30) Dharmalingam, S. Biodegradation and photodegradation of 621 polylactic acid and polylactic acid/polyhydroxyalkanoate blends non- 622 woven agricultural mulches in ambient soil conditions. Ph.D. 623 Dissertation, University of Tennessee, Knoxville, TN, 2014.

(31) Ludueña, L.; Vázquez, A.; Alvarez, V. Effect of lignocellulosic 625 filler type and content on the behavior of polycaprolactone based eco- 626 composites for packaging applications. Carbohydr. Polym. 2012, 87, 627 411-421.

(32) Rocca-Smith, J. R.; Chau, N.; Champion, D.; Brachais, C. H.; 629 Marcuzzo, E.; Sensidoni, A.; Piasente, F.; Karbowiak, T.; Debeaufort, 630 F. Effect of the state of water and relative humidity on ageing of PLA 631 films. Food Chem. 2017, 236, 109-119. 